\section{AUFGABENSTELLUNGEN UND METHODEN}

In diesem Kapitel sollen die zentralen Aufgaben, die Vorgangsweisen zu deren Enfüllung sowie die danin verwendeten methodischen Werkzeuge kurz edäutent werden.

\subsection{Aufbau eines Metadatenkataloges}

Ziel der Studie war unter anderem die Erstellung einer intemetfähigen Fakten- und Datensammlung zum physiographischen, ökologischen, sozioökonomischen und kulturellen Status der Biosphärenparks. Neben der Bereitstellung von Basiswissen sollen diese Informationen auch in der Forschungsplanung und in der Diskussion eines dem Biosphärenparkkonzept entsprechenden Forschungsprogramms Anwendung finden. Als Zielgnuppen sind das MAB-Nationalkomitee, die Biosphärenparkmanager und die Forscher einerseits sovie die breite Öffentlichkeit und Interessiente andererseits definient.

Die Vorgaben fanden in der Erstellung eines Metadatenkataloges einen Niederschlag. Unter Metadaten (Daten über Daten) versteht man strukturierte Informationen, mit deren Hilfe eine Informationsressource beschrieben und dadurch besser auffindbar gemacht wird. Auf die Abstimmung mit den vorgegebenen Strukturen des MAB-Programms uurde bei der Aufbereitung der Daten besonderer Wert gelegt.

Der Aufbau eines Metadatenkataloges enfordert folgende Schnitte:

- Sichtung von Fachliteratur zu Metadatenkatalogen oder-banken

- Abklänung möglicher Inhalte für die Datenbankstruktur entsprechend den Vorgaben und Zielgnuppen

- Enturuf eines Datenbank-Designs

- Rücksprache mit dem Auftraggeber

- Auswahl der technischen Umsetzungsinstrumente

- Konzept zur Vemetzung und Gewichtung von Inhalten

- Konzept zur Beschlagwortung
- Literatur- und Untenlagenrecherche übers Intemet und über Intervieus

- Literatur und Untedagenauswahl entsprechend der Inhaltsdefinition

- Einarbeitung der Untedagen anhand Informationen aus der Recherche

- Vervollständigen der Datensätze durch Sichtung der Untedagen jeweils vor Ort (Entlehnstellen)

- Design einer Abfrageoberfläche

- Statistische Auswertung der Einträge

- Evaluienung

\subsubsection{Allgemeine Merkmale}

- Datendrehscheibe: Die Metadatenbank ist keine Sammlung von Information. Sie ist eine Sammlung von Daten, die Zugang zu Information emöglichen. In diesem Sinne sollte ein Schwenpunkt der Metadatenbank darin liegen, vor allem Verweise auf Sammeldaten bereitzustellen. So ergibt es keinen Sinn, tausende gesammelte Werke der Bibliothek der Forschungsstation Illmitz in die Datenbank einzugeben. Vielmehr soll die Station selbst in der Metadatenbank als eine zentrale Informationssammelstelle für den Biosphärenpark Neusiedler See ersichtlich gemacht werden.

- Breites Spektrum an Datenarten: Die vorliegende Metadatenbank muss ein hohe Anzahl verschiedenartiger Datenarten verarbeiten. Diese Daten reichen von Berichten über Kartenwerke, Informationssysteme, Datensammlungen bis hin zu Organisationen. Diese Heterogenität stellt hohe Anfordenungen an die Datenbankstruktur, damit alle Datenarten ausreichend und verständlich erfasst werden können.

- Metadatenbank als „never-ending story“: Die Metadatenbank ist als eine laufend zu erweitemde Sammlung von Daten über Daten zu sehen. Die vorliegende Recherche leistet einen wesentlichen Anteil an der Sichtung biosphärenparkrelevanter Daten, sie kann jedoch nicht 
alle relevanten Daten bereitstellen. Vielmehr stelt sie eine Basis zur Verfügung, die erst durch kontinuiediche Weiterentwicklung über die Jahre die entsprechende "Reife" erreichen kann. Die Praxistauglichkeit steigt mit der Anzahl der Einträge. Anspruch auf Vollständigkeit kann, wie naturgemäß bei allen Arten von Metadatenbanken, nicht geleistet werden.

- Intemetfähige Aufbereitumg: In Rücksprache mit dem Auftraggeber wird die Metadatenbank als Acoess-Datenbank bereitgestellt. Die Umsetzung als eine webbasierte Anwendung ist in diesem Rahmen nicht leistbar. Es sollen aber jene Schnittstellen geschaffen werden, die für eine Umsetzung vonnöten sind.

\subsubsection{Technische Merkmale}

\section{Beschreibungskriterien}

Zur Identifizienung und Beschreibung eines Datensatzes sind wenige, aber eindeutige Kriterien fest zu legen. Anhand der Access-DatenbankEingabeoberfläche werden im Folgenden die Eingabemöglichkeiten edäutert.

Die Eingabeoberfläche besteht aus einem Formularkopf und drei Unterformularen. Am Fommularkopf werden zunächst die ID und das Datum der Eingabe automatisch vergeben. Hier erfolgt auch die Eingabe des Titels, des Objekttyps, der Objektsubtyps, der räumliche Ausdehnung und ev. der Aktualität. Die weiteren Eingabekriterien sind in folgenden Abbildungen kurz dargestellt.

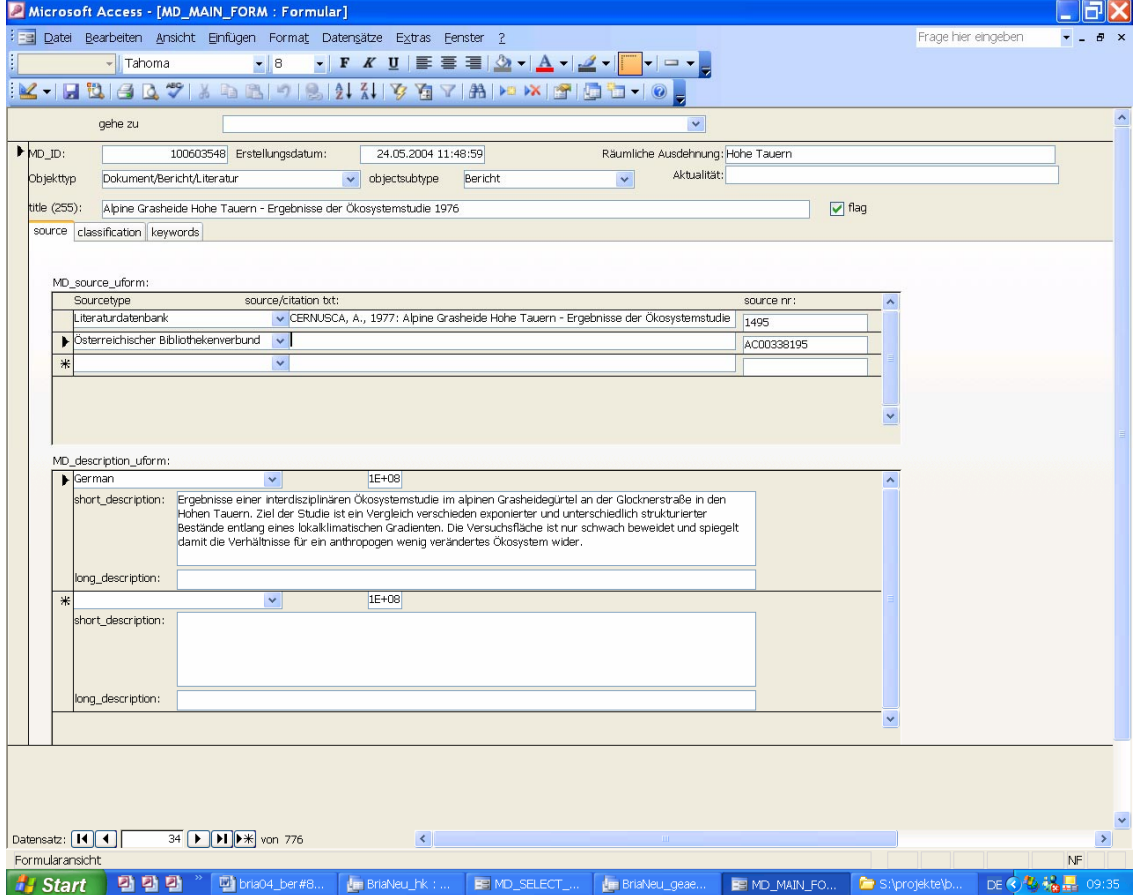

Abbildung6: Madadatenbank-Einggbedbeflädhe,,saurce".

Auf der Eingabesite „saurce" efolgen die Eingabe des Zitats und der Datenqudle und eine Kurzbeschreibung 


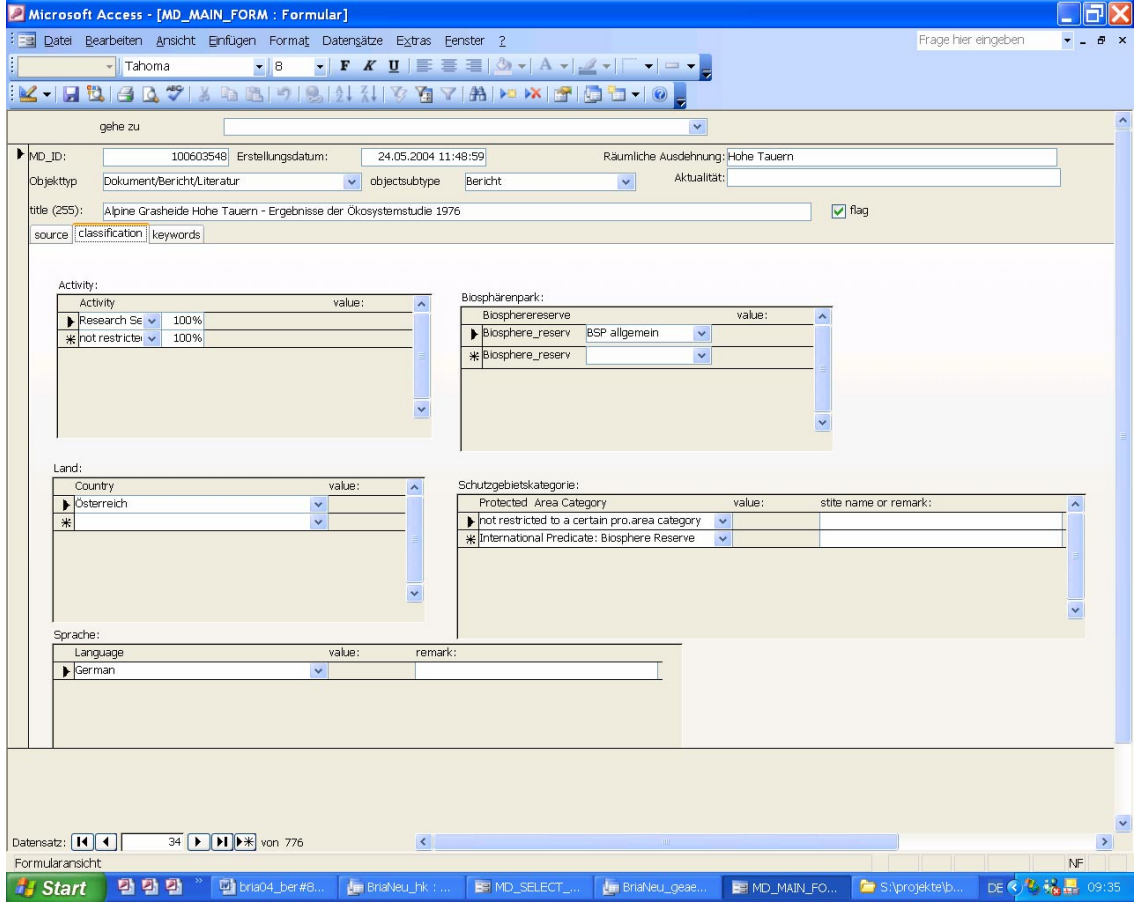

Abbildung7: MAadatenbank-Einggbedbefläche,,dassification“.

A uf der Eingabesate „dassification“ efdyt die Eingabe von Methoden zum Managenent von Schutzgdiden (Todse genäß Projkt IPAM), die Eingabe des Landes der Sprache der Zuordhungzu einemBiosphärenpark und dieSchutzkategrie, auf diesich der Eintragbeziett.

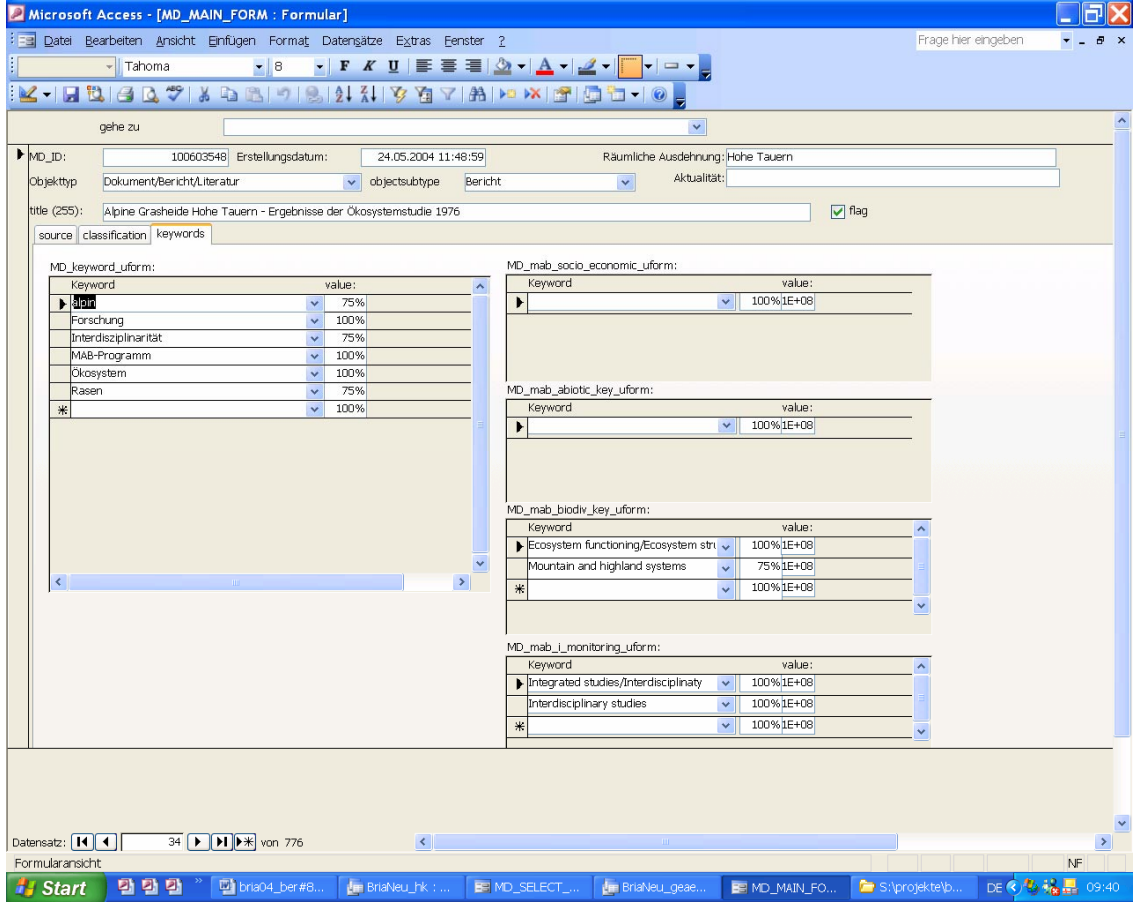

Abbildung8: Matadatenbank-Eingabedbeflähe,,keyward".

Auf der Eingabescite ,kgenard" efdyt die Beschlagnatung des Datensatzes anhand der zwe vervendten Schlagnatlisten

\section{Beschlagwortung}

Die Beschlagwortung effolgte nach zwei Schlagwortkatalogen:

MAB-Schlagmontliste: Die Einträge werden gemäß MAB-Schlagwortliste (vergl. UNESCO. THE MAB PROGRAMME) getätigt. Der zweistufige Katalog (vier Hauptthemen mit jeweiligen Unterthemen) ist auf die globale Ebene zugeschnitten und dementsprechend unspezifisch in seiner Einteilung. 


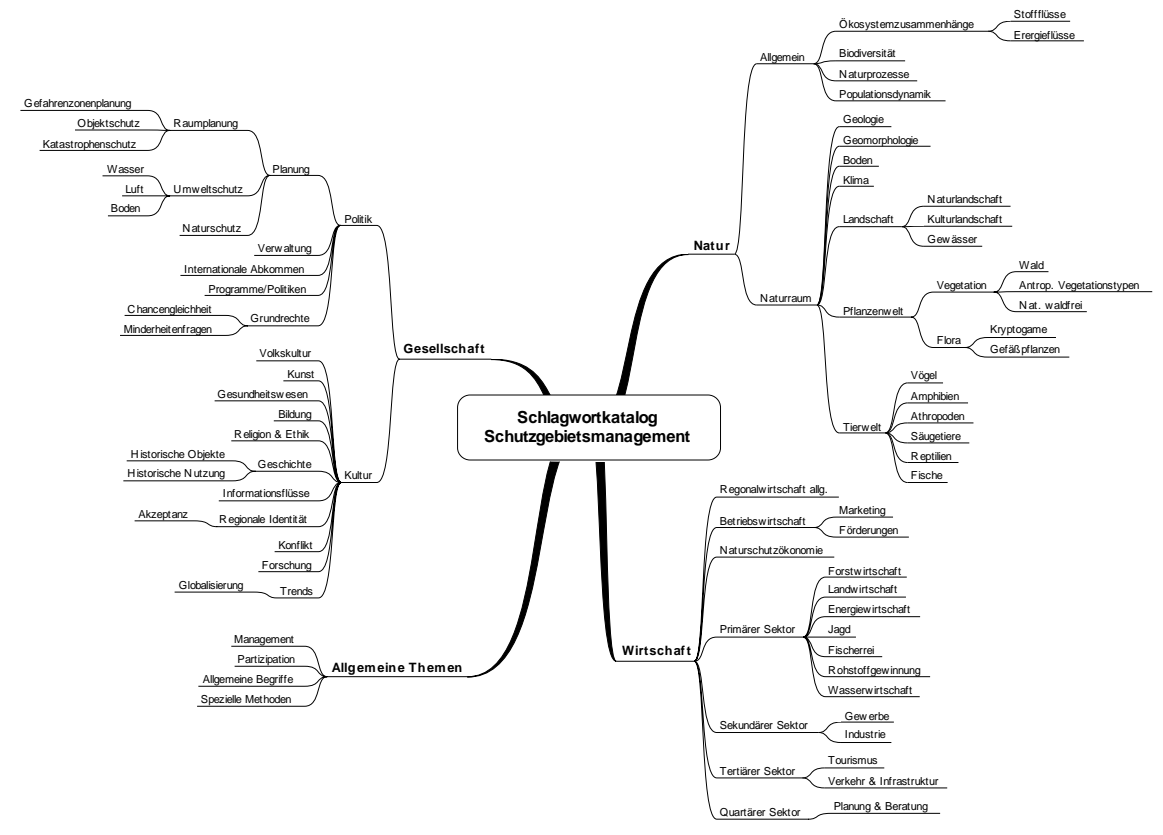

Abbildung9: Hierardischer Sdhagnatbaum

Der dargestelte Schlagnatbaum stelt de doern Ebenen der Hierarchie dar. Die lezte Ebene kann aufgund der V idzahl an Schlagnaten nidht merr in dieser Formskizziet weden Stand 20-10-2004.

Hierarchischer Schlagmortbaum Zum anderen wurde ein vielstufiger hierarchischen Schlagwortkatalog (vergl. Abbildung 9) entworfen, der gegenüber der MAB-Schlagwortliste besser auf die großsmaßstäblichere nationale Ebene abgestimmt ist. Die Beschlagwortung erfolgt dabei anhand der Schlagworte der letzten (4.) Hierarchiestufe. Da die Schlagworte unter den Hierarchiestufen mit einander verknüpft sind, kann bei der Suche durch den Benutzer optional eine beliebige Hierarchiestufe ausgewählt werden. Des Weiteren kann den einzelnen Schlagnöntem ein Wert für die Relevanz (z.B. $100 \%, 75 \%, 50 \%$ oder $25 \%$ zugeordnet werden, der eine zutreffendere, gewichtete Beschlagwortung des Datensatzes zulässt.

\section{Datenbankstruktur}

Die Datenbank ururde als relationales Datenbankmodell konzipient. Unterschiedliche Beschreibungseinheiten (Entitäten) urrden in eigene Tabellen gefasst und diese miteinander verknüpft. Die zentrale Tabelle mit der Bezeichnung „METADATA“ beinhaltet die Metadatenobjekte. Jedes Metadatenobjekt ist durch verschiedene Attribute charakterisient. Da oft mehre Attributausprägungen für ein Metadatenobjekt zutreffen, wurden die meisten Attribute in eigenen Untertabellen enfasst. Um der Anfordenung Mehrsprachigkeit zu entsprechen, wurden die Attributausprägungen in Form von Code-Zahlen gespeichert. Die „Übersetzung“ dieser Codezahlen effolgt mit eigenen Verweistabellen.

Beispiel: Eine Publikation ist ein Metadatenobjekt. Die Quelle (Zitat, Bibliothek) wird in der Tabelle MD_SOURCE abgespeichert und erhält eine eindeutige Nummer (MD ID). Eventuell kann die Publikation auch digital im Intemet verfügbar sein, dann gibt es einen zweiten Eintrag in der MD SOURCE Tabelle zu diesem Metadatenobjekt. In ähnlicher Weise können auch die anderen Attribute (Biosphärenpark, biogeografische Region, Land, (Kurz-, Lang-Beschreibung) mit einem oder mehreren Einträgen beschnieben verden. Die Beschlagwortung exfolgt zweifach, einmal gemäß der MAB-Schlagwortliste und einmal mit der umfassenden, hierarchischen Schlagwortliste. 


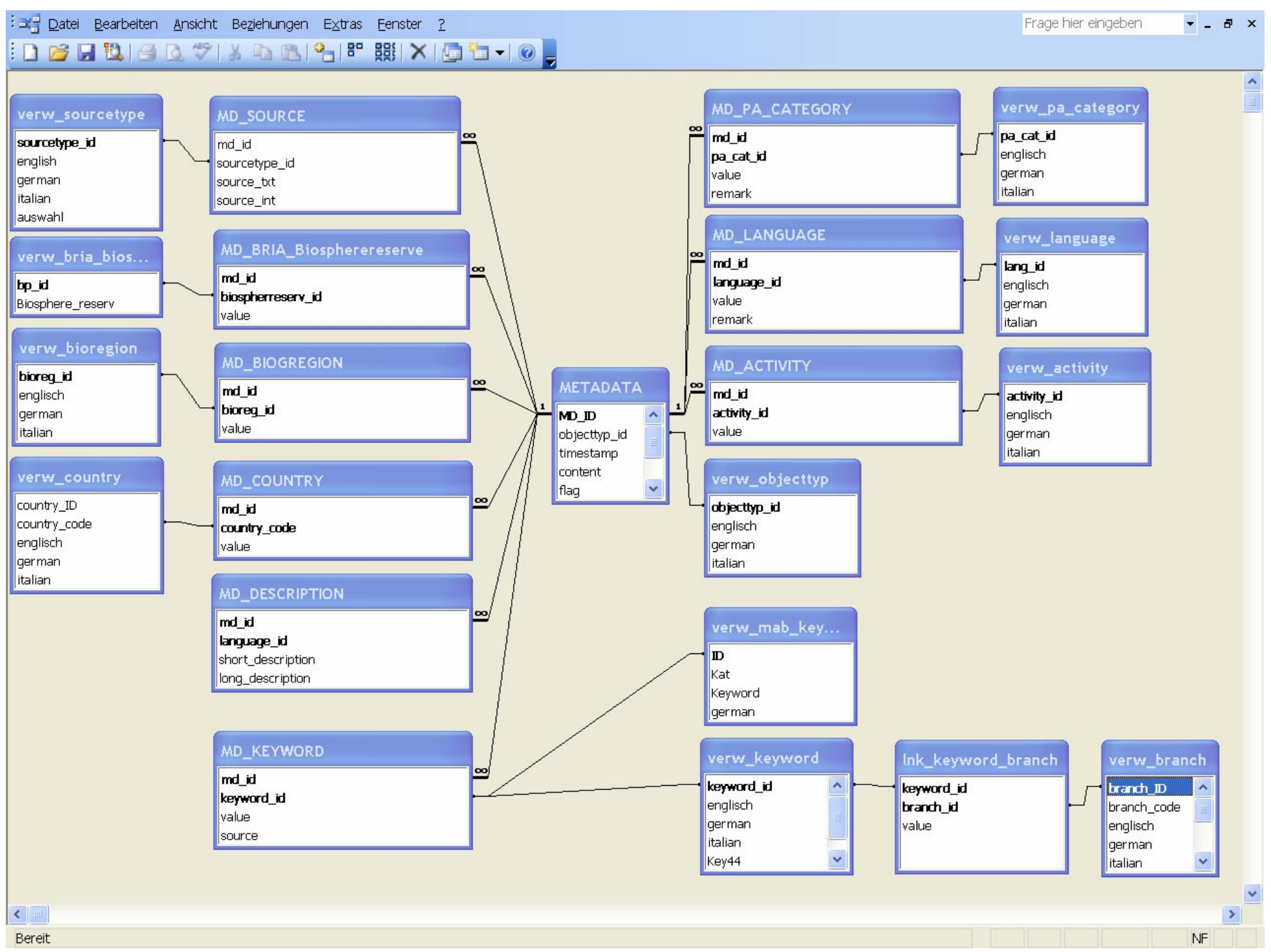

Abbildung10: ER-Diagrammder Datenbankstruktur für den Mtadatenkatalog 


\subsection{Analyse Forschungsstand und Forschungsbedarf}

Ziel dieses Arbeitschnittes war die Erstellung einer Übersicht über Forschungsaktivitäten in den österreichischen Biosphärenparks. Die Studie soll die bisherige Forschung in und über österreichische Biosphärenparks (inklusive Planungsgebiet Wienerwald) in einer leicht enfassbaren Form dokumentieren. Ergebnisse der östemeichischen MAB-Forschung außerhalb der Biosphärenparks, aber mit Relevanz zur Biosphärenparkforschung sollen ebenfalls berücksichtigt werden.

Der potenzielle Biosphärenpark Moravien (Planungsarbeiten vorübergehend eingestellt) konnte nicht bearbeitet werden, da der Zugang zu Daten ungenügend ist und es an Ansprechpartnem mangelt.

Konzept zur Analyse des Stands der Forschung: Das grundsätzliche Problem liegt darin, eine Fülle von oft unübersichtlichen und miteinander in Beziehung stehenden Daten zur Forschungslandschaft in komprimierter und leicht verständlicher Weise darzustellen. Die konzeptionellen Überlegungen von JUNGMEIER \& HAUSHERR (1998) bZW. RECKENDORFER et al. (1998) geben hienfür eine Struktur vor, die zur Analyse des Forschungsstands sehr gut geeignet erscheint.

Das hier leicht abgewandelte Konzept sieht vor, die Forschungslandschaft anhand von vier Leitfragen in ihren wichtigsten Facetten abzubilden. Den Leitfragen werden Kriterien zugeordnet, die zur Beantwortung dieser Leitfragen herangezogen werden:

- WIEVIEL? - Stellenwent der Forschung: Aus der Fülle der Forschungsaktivitäten lassen sich Rückschlüsse auf den Stellenwert der Forschung im Gebiet des Biosphärenparks ziehen.

Kniterien:

- Aktuelle und abgeschlossene Forschungsprogramme/ -aktivitäten

- Geplante Forschungsvorhaben

- Ressourcenausstattung Sponsoring)
- Vergleich mit der Umgebung/ mit anderen Biosphärenparks

- Intemationaler Kontext

- Forschungsinfrastrukturen

- Forschungskonzepte

- WOZU? - Zweck und Ziele der Forschung: Forschungszielen und Forschungsarten sollen herausgearbeitet werden. Sie können je nach Einbettung der Forschung (z.B. Nationalparkverwaltung, Universität) unterschiedliche Ausprägungen haben. Die Ziele können generell auf der Ebene des Biosphärenparks angesiedelt sein oder sich auf spezielle Forschungsaktivitäten beziehen.

Kriterien:

- Forschungskonzepte

- Forschungsziele

- Nutznießer/ Stoßnichtung (Forschungsarten)

- Bezug zur MAB-Forschung

- Einbeziehen der Allgemeinheit

- Öffentlichkeitsanbeit

- WAS? - Inhalt und Gegenstand der Forschung: Diese Leitfrage nimmt starken Bezug auf das disziplinäre Design, die thematischen Schwerpunkte und die zu bedienenden Leitfragen oder Forschungsfelder.

Kriterien:

- Forschungsdisziplinen

- Leitfragen und Forschungsfelder

- Themenschwerpunkte

- Räumliches und zeitliches Design

- WIE? - Organisation und Ablauf der Forschung: Sie sind in vieledei Hinsicht den Rahmenbedingungen und bestimmten Ablaufmechanismen unterworfen. 
Kniterien:

- Öffentlichkeitsarbeit

- Forschungskonzepte

- Aufteilung der Forschungskompetenzen (zentral/ dezentral)

- Intemationaler Kontext

- Kooperationen

- Forschungsinfrastrukturen und Forschumgsdokumentation

- Finanzienung

Konzept zur Analyse des Forschings- und Handlungsbedarfs: Forschung ist ein wesentlicher Teil der Biosphärenparkidee und kann daher nicht losgelöst davon, sondem nur im Gesamtzusammenhang betrachtet werden. In diesem Sinne ist auch die Analyse des Forschungs- und Handlungsbedarfs zu sehen, die versucht anhand von Forschungsdeteminanten (Defizite und Probleme, Schwenpunkte und Potenziale) die grundsätzlichen Bedingungen für das Funktionieren der Biosphärenparkforschung zu bestimmen. Detaillierte Festlegungen (konkrete Forschungsfelder, Themenschwerpunkte etc.) können nur im Zuge regionaler, partizipativer Prozesse enfolgen. Anhand einer Mind Map wird versucht, die Forschungsdeterminanten übersichtlich darzustellen und daraus den Forschungs- oder Handlungsbedarf abzuleiten.

Datengrumdlagen zur Analyse: Die Ergebnisse zum Status quo der Forschung kommen durch die Auswertung von vier Quellen zustande:

- Standardisierte Experteninterviews: In Absprache mit dem Auftraggeber wurden 14 Interviewpartner zum Thema Forschung ausgewählt (vergl. Anhang-5). Ziel war es, vor Ort tätige Forscher zur regionalen Forschung $\mathrm{zu}$ befragen. $\mathrm{Zu}$ diesem Zwecke wurde ein standardisierter Fragebogen mit 26 Fragen (A bis Z) - abgestimmt auf die Leitfragen - erstellt und den Expenten vorab zur Durchsicht zugesandt (vergl. Anhang-3). Die nachfolgenden Interviews mit den Experten dauerten zwischen 1,5 und 3 Stunden.

- Literaturauswertung: Die Literaturrecherche ergab rd. 1500 Datenobjekte. 781 davon kamen in eine engere Auswahl und wurden damit in die Datenbank aufgenommen. 620 Datensätze sind vollständig eingegeben und bilden den Stand des Metadatenkataloges vom 20.10.2004. Die restlichen 161 Datensätze werden in der Datenbank evident gehalten, meist fehlen nur Teilaspekte. Nach einer weiteren Recherche zur Ergänzung fehlender Details können diese Datensätze ebenfalls in den Metadatenkatalog überführt werden. Anhand statistischer Auswertungen konnte Einblick über abgeschlossene und aktuelle Forschungsaktivitäten gewonnen und in die Analyse eingebaut werden. Viele Werke (vor allem histonische Arbeiten) scheinen in Online-Katalogen nicht auf und konnten daher nicht erhoben werden. Eine vollständige Literaturauswertung vürde durch die Vielzahl an dezentral verwahrten Dokumentationen, durch eingeschränkten Zugniff aufgrund bürokratischer Vorgaben oder eingeschränkte Online-Verfügbarkeit den Rahmen dieser Studie sprengen.

- Workshopergebnisse: Der Biosphärenparkmanager-Workshop vom 27. Mai 2004 in Wien sowie der intemationale MAB-Consultancy Workshop vom 17. bis 18. Juni in Illmitz brachten weitere Aspekte aus Sicht der Region (bottom-up) sowie intemationaler Vorgaben (top-down) zum Vorschein (vergl. Anhang-6).

- Gutachtliche Einschätzung des Bearbeiterteams: Die aus langjähnigen Erfahnungen des Bearbeitenteams resultierenden Kenntnisse und die Experteneinschätzung aus den Interviews sind in ergänzender und verfeinemder Weise in die Analyseergebnisse eingeflossen.

Darstellungsformen: Die Darstellung der Ergebnisse enfolgt aufgnund der unterschiedlichen Bezugsquellen und der stark heterogenen Daten überwiegend qualitativ. Wo möglich, uurde die Darstellung grafisch mit Hilfe von Spinnendiagrammen oder Barometer-Balken gelöst (vergl. Abbildung 11, Abbildung 12 ).

Abbildung 11 zeigt die Darstellungsfomm fïr die Forschumgsarten Grundsätzlich können drei Forschungsrichtungen unterschieden verden (vergl. JUNGMEIER \& HAUSHERR 1998):

- Allgemeine Gnundlagenforschung: Unter dieser Kategorie vird die Enforschung wissenschaftlicher Fragestellungen, die nicht 
schutzgebietsspezifisch sind, verstanden

- Regionsbezogene Inventare und Analysen: Dokumentationen zum Ist-Zustand der Region, z.B. Kartienungen, Haushaltsanalysen etc. fallen unter diese Kategonie.

- Begleitforschung fürs Management/ angewandte Forschung: Danunter sind Forschungen zur Klärung schutzgebietsspezifischer Fragen wie Entwicklung und Evaluienung/ Monitoring von Maßnahmen zu verstehen.

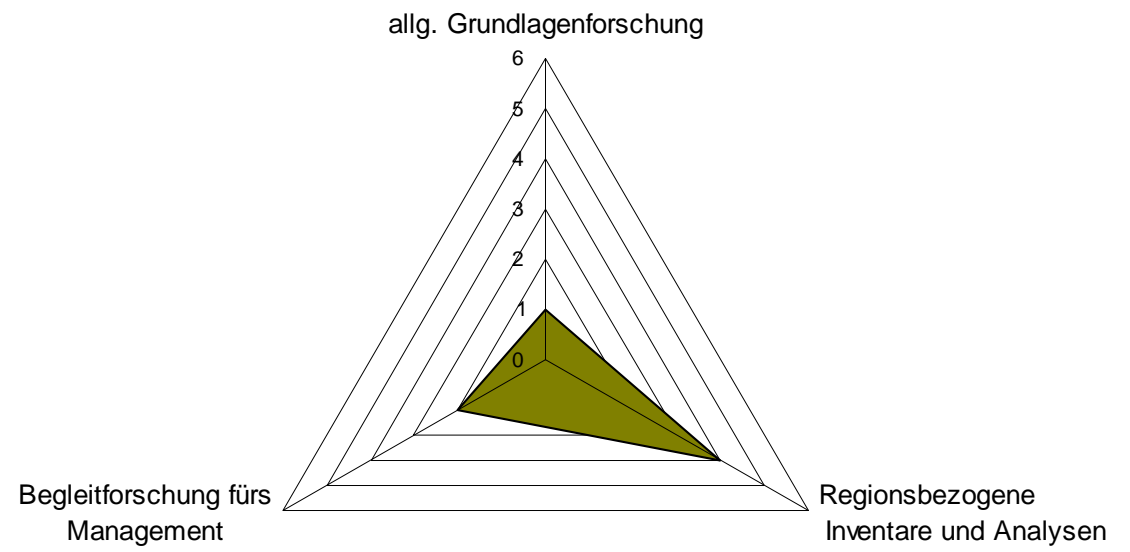

Abbildung 11: Beispidsdiagrammzur Forschungsart.

Nach der Auspägung des günen Dreieks entlang der jeneilign Adøen ist die Schwerpunktstzung dar Faschung zu benteilen Imkonkreten Beispid (Großes Walsetal) sind regionsbezogenen Inventare und Analysen die vorviegenden Forshungsarten in der Region (Auspäang 4). Eine Vedeich der Diagramme dr einzdnen Biosphärenparks ist nur eingeschränkt mögich

Abbildung 12 zeigt, ebenfalls am Beispiel Großes Walsental, die Darstelhungsfomm für die Forschumgsdisziplinen. Grundsätzlich können sechs Disziplinen unterschieden werden:
- Naturwissenschaften - biotisch

- Naturwissenschaftlen - abiotisch

- Geistes- und Sozialwissenschaften

- Technische Wissenschaften

Wirtschaftswissenschaften

- Interdisziplinäre Wissenschaften

Unter interdisziplinären Wissenschaften sind jene zu verstehen, die hinsichtlich ihrer Forschungsziele und Forschungsagenden mindestens zwei der oben genannten Richtungen als gleichwentige Partner verstehen.

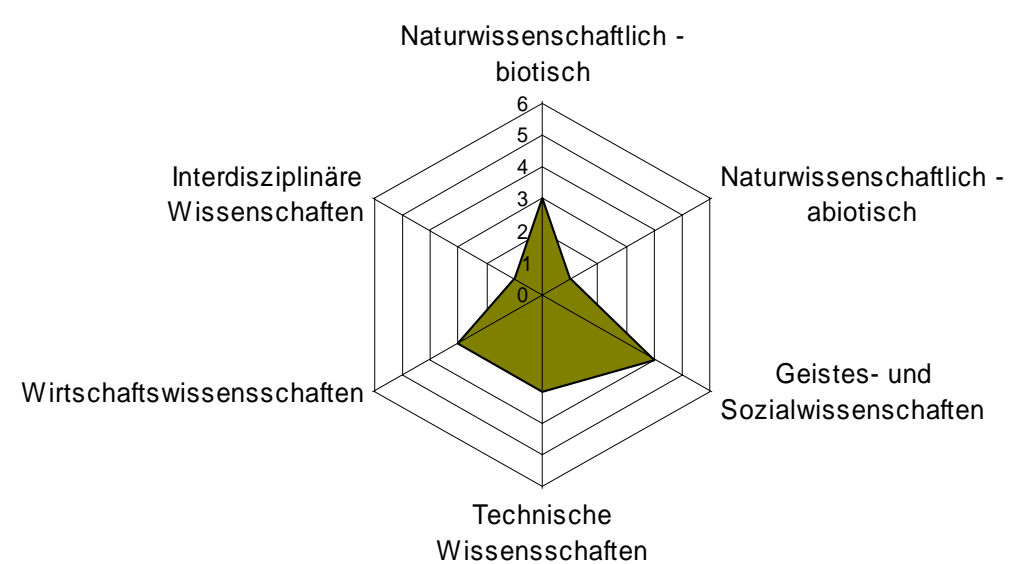

Abbildung12: Beispidsdiagrammzu den Forshunosdisziplinen

Auch hier ist de Schmepunktstzung nach der Ausprägung des günen Dreieks entlang de jeveiligen A dsen zu beneten ImBeispid Großes Walsetal ist keineauffallendeDoninanz eine eirzdnen Disziplin zu ekennen Dieses Diagrammlässt sich als rdativ geichmäßige V etailung über die Disziplinen hirmeg intepptieen mit leidtem Sdmenunkt auf den Geistes und Sovialvissenshaften und einem doch dautlichen Fehlen von intedisziplinären Forshungsansätzen EineV edjeich der Diagrammeder einzdnen Biosphärenparks ist nur eingeshränkt mögich

Weitere Aspekte, die bei der Interpretation der Ergebnisse zu 
berücksichtigen sind:

- Auswahl der Intenviewpartner für Expenteninterviews: In Absprache mit dem Auftraggeber (Östemeichisches MABNationalkomitee, Prof. Grabherr) urrde versucht, Forscher(gruppen) auszuwählen, die intensive MAB-relevante Forschungsarbeit in den Biosphärenparks bzw. den zugehönigen Regionen leisten. Der aktuelle Forschungsstand führte zu einer Konzentration auf die naturvissenschaftlichen Disziplinen.

- Die Expentenintenviews stellen die subjektive Sichtweise von Fachleuten dar, die über gute Kenntnisse zur regionalen Forschung verfügen. Eine objektive Bewertung ist daraus nicht ableitbar.

- Keine spezifische Biosphärenparkforschung vorhanden: Bei den Analysen ist anzumerken, dass es sich dabei jeweils auf die Forschungsaktivitäten in ihrer Gesamtheit bezieht (sämtliche Forschungsaktivitäten sind Ziel der Erhebung), da spezifische Biosphärenparkforschung in Österreich bis auf wenige Ausnahmen fehlt.

- Visualisienung der Kemaussagen: Die Diagramme sollen in Art Herkunft, Qualität und Quantität äußerst heterogene Daten auf einen Blick enfassbar machen. Ihnen liegt somit keine oder nur teilweise statistische Genauigkeit zugnunde, vielmehr findet eine starke Verdichtung der Eingangsparameter statt (Komplexitätsreduktion auf ein Modell). Informationsverluste sind dabei unausweichlich. Die gewählte Form der Visualisienung wird jedoch als goeignetes Mittel angesehen, um Kemaussagen übersichtlich darzustellen.

- Eindringtiefe: Die gewählte Eindringtiefe ist vor dem Hintergnund der nur ansatzweise implementierten Biosphärenparkidee, dem damit einhergehenden Manko an Unterlagen und Anknüpfungspunkten und fehlenden auf Österreich abgestimmten Evaluienungsmethoden zu sehen. $\mathrm{Zu}$ detaillierte Aussagen hätten nur beispielhaften oder zufälligen Charakter gehabt. Ziel war aber die Generienung einer Gesamtsicht, die kare, nichtungweisende Aussagen über grundsätzliche und dringlich zu behandelnde Eckpunkte/ Basisfunktionen/ Enfordemisse treffen lässt.
- Evaluienumg der Ergebnisse: Aus den Unschärfen der vorhandenen Daten und subjektiven Ansichten der involvienten Personen eine fundiente Gesamtsicht mit dem Anspnuch wissenschaftlicher Genauigkeit zu erstellen, ist mit gevissen Unsicherheitsgraden behaftet. Es wird daher folgender Ansatz gewählt: „Data don't have to be exact, but all have to agree upon". Gegen Projektende werden daher die im Projekt involviesten Personen gebeten, den Berichtsentwurf nochmals mit ihren Ansichten abzugleichen.

- Berücksichtigung unterschiedlicher Entwicktungsstufen: Die Bewertung hat den unterschiedlichen Entwicklungsstufen, in denen sich die Biosphärenparks befinden, Rechnung zu tragen. Eine ähnliche Ausprägung eines Kriteriums in zwei Biosphärenparks kann daher trotzdem zu unterschiedlicher Bewertung führen.

\subsection{Analyse der Implementierung der Sevilla- Strategie}

Ziel dieses Abschnittes ist es, die 1995 in Sevilla/ Spanien im Zuge einer intemationalen Expertenkonferenz erarbeitete Sevilla-Strategie zu überprüfen. Diese Rahmennichtlinie führt konkrete Maßnahmen, Schnitte und Indikatoren an, denen für die weitere Entwicklung der Biosphärenparks/ des Biosphärenparknetzes im 21. Jahrhundent maßsgebliche Bedeutung zugemessen wird.

Bewertungskonzept: Die Sevilla-Strategie weist einen stark umsetzungsorientienten Charakter auf. Je mehr Aktivitäten ein Biosphärenpark setzt, desto mehr Kriterien sind auch anwendbar. Im Gegensatz dazu weisen Biosphärenparks ohne emsthafte Umsetzungsanstrengungen nur wenige Anknüpfungspunkte auf. Eine Überprüfung reduzient sich folglich auf einige wenige Kriterien. In Anbetracht der eingeschränkten Biosphärenparkaktivitäten in Östemeich liegt es daher nahe, zunächst die prinzipielle Eignung der Biosphärenparks für diese Schutzgebietskategorie zu überprüfen. Die Enfüllung von Mindestkniterien ist Voraussetzung dafür, dass die Sevilla-Richtlinien 
überhaupt zur Anwendung kommen können. Es wird, auch im Hinblick auf ein fehlendes Übenprüfungskonzept auf nationaler Ebene, folgende zweistufige Vorgangsweise gewählt:

1. Überprüfung der grundsätzlichen Eignung zum Biosphärenpark

2. Überprüfung der Indikatoren entsprechend der Sevilla-Strategie

Überprüfung der grundsätzlichen Eignung zum Biosphärenpark: Unter zu Hilfenahme der in Deutschland zur Überprüfung der Anerkennung erarbeiteten Ausschlusskniterien (vergl. UNESCO 1996) wird zunächst die prinzipielle Eignung zum Biosphärenpark in Form eines Balken-Barometers dargestellt (vergl. Abbildung 13). Diese Mindestkniterien sind auch in der Sevilla-Strategie selbst integriert, ihre Aussagekraft wird jedoch durch eine separate Betrachtung ehöht.

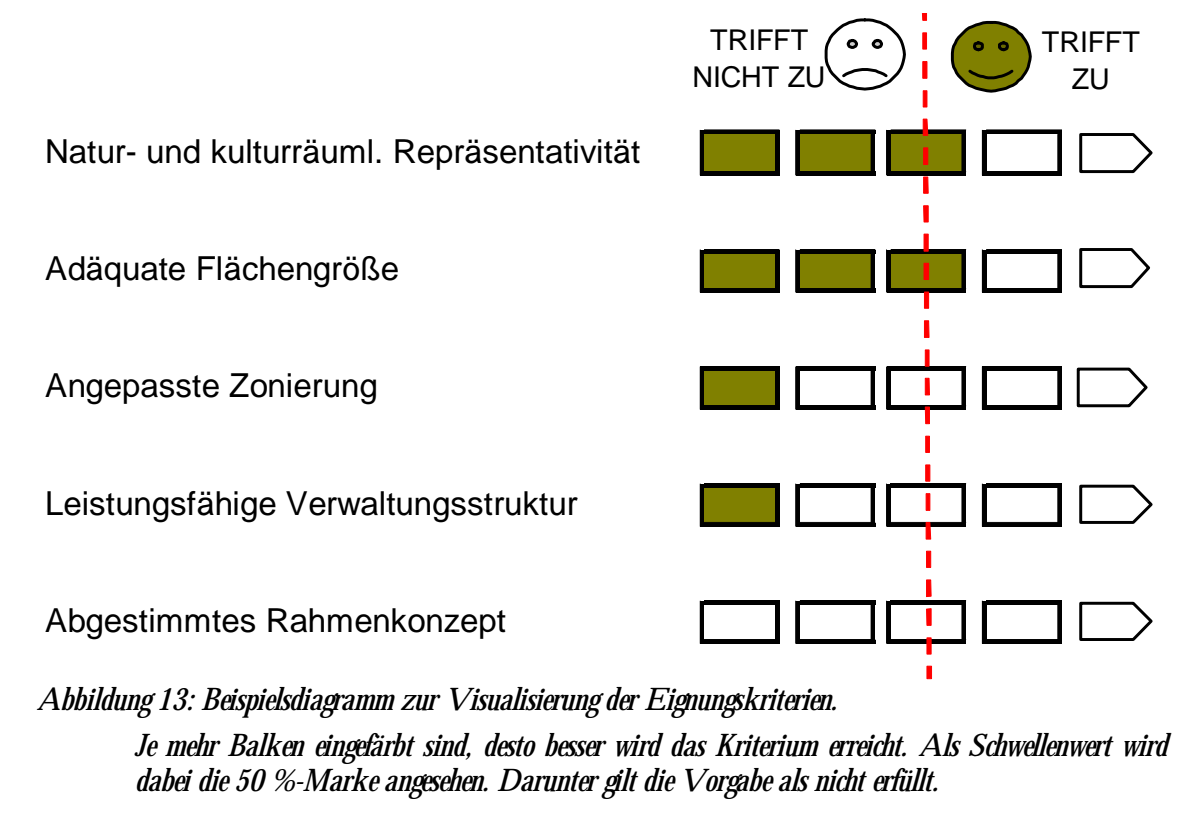

Naturr- und kultumäumliche Repräsentativität: Ein Biosphärenpark soll Ökosystemkomplexe aufweisen, die von anderen Biosphärenparks in
Österreich nicht ausreichend vertreten werden. Es soll auch benücksichtigt werden, invieweit die Ökosystemkomplexe Interaktionen zwischen Mensch und Natur zulassen. Bei der Beurteilung ist außerdem zu beachten, ob dieser Ökosystemkomplex nicht bereits durch eine andere Schutzkategonie hinreichend abgedeckt wird.

Die Bewertung kann auf Grundlage der Kulturlandschaftskartienung Österreichs (vergl. WRBKA \& FINK, 1997) getroffen werden, die speziell für diese Zwocke ein sehr gut goeignetes Instrumentanium darstellt.

Adäquate Flächengröße: Eine gevisse Mindestflächengröße ist Voraussetzung, um die drei Gnundfunktionen von Biosphärenparks, die Schutz-, Entwicklungs- und logistische Funktion, effüllen zu können.

Die in Deutschland mit 30.000 ha angegebene Mindestgröße scheint für Östemeich aufgnund der hier überwiegend keinstrukturienten Landschaft nicht sinnvoll. Daher wird keine fixe Mindestgröße angegeben. Es soll vielmehr die Definition einer geschlossenen Landschaft gelten, die dem allgemeinen Verständnis nach als Region bezeichnet werden könnte. Kennzeichnend für eine Region ist, dass sie in ihrer Funktion als Natur-, Lebens-, Wirtschafts- oder Erholungsraum als Einheit mit intensiven regionalen Verflechtungen wahrgenommen wird.

Angepasste Zonienung: Der Biosphärenpark muss in Kem-, Pflege, und Entwicklungszone gegliedert sein.

Die in Anlehnung an die Vorgaben der UNESCO für Deutschland angepassten Zonienungsanteile lauten wie folgt:

- Kemzone: mindestens 3\% der Gesamtfläche

- Pflegezone: mindestens $10 \%$ der Gesamtfläche

- Kem- und Pflegezone zusammen: mindestens $20 \%$ der Gesamtfläche

- Entwicklungszone: mindestens $50 \%$ der Gesamtfläche

Aufgrund fehlender nationaler Vorgaben wird diese Einteilung zur Bewertung herangezogen. Sie sind auch für österreichische Verhältnisse gut anvendbar.

Leistungsfähige Verwaltungsstmuktur: Das Management muss über das für einen Biosphärenparkbetrieb gemäß intemationaler Leitlinien 
notwendige Verwaltungs- und Fachpersonal und über entsprechende Sachund Finanzmittel verfügen. Die Verwaltung sollte innerhalb von drei Jahren vollständig etablient sein.

Exakte Vorgaben können bei diesem Kriterium nicht gemacht werden. Hier ist die Bewertung des vorhandenen Managements ausschlaggebend.

Abgestimmtes Rahmenkonzept: Ein umfassendes Rahmenkonzept (Leitbilder-> Teilziele-> Umsetzungsstrategien-> Evaluienung; Finanzplan) für Schutz, Pflege und Entwicklung soll innerhalb von drei Jahren unter breiter öffentlicher Einbindung erarbeitet werden.

Neben der Analyse vorhandener Dokumente und Untedagen wird auch hier die Bewertung des vorhandenen Biosphärenparkmanagements als ausschlaggebend betrachtet.

\section{Überprüfung der Indikatoren entsprechend der Sevilla-Strategie:}

Anhand des von der UNESCO erarbeiteten „,Survey on the Implementation of the Seville-Strategy" wird versucht, in Expertenintervieus entsprechende Hinweise auf den Implementienungsgrad auf Biososphärenparkebene zu ehalten. In Kombination mit einer Literaturauswertung und der gutachtlichen Einschätzung des Bearbeiterteams soll dadurch ein Gesamtbild entstehen. Entsprechend den vier Hauptzielen der Sevilla Strategie enfolgt die Auswertung (vergl. Abbildung 14):

- Use Biosphere Reserves to oonserve natural and cultural diversity (,maintenance of natural and cultural diversity"): Zwar bestehen auf Biosphärenparkebene keine Empfehlungen, eingeschränkt sind jedoch die Empfehlungen auf nationaler Ebene auf die Biosphärenparkebene übertragbar. Beispielhafte Kriterien: In-situ Artenschutz, Wiedereinbürgenungen, Ökosystemrehabilitienungen, grenzüberschreitende Kooperationen, etc.

- Utilise Biosphere Reserves as models of land management and of approaches to sustainable development („,models of land management and sustainable development"). Beispielhafte Kriterien: Stakeholder mapping, Partizipation, Produktentwicklung Produktlabelling, Anreizsysteme zum sparsamen Umgang mit Ressourcen, Maßnahmen zur Erhöhung der regionalen Wertschöpfung, Instrumentarien zum
Interessensausgleich, Entwicklung von Best Practice, Überprüfung bestehender Landnutzungsweisen und Stoffströme, Leitbildentwicklung, Berücksichtigung globaler Trends, Aufbau regionaler Netzwerke, etc.

- Use Biosphere Reserve for research, monitoning education and training (,,research, monitoning, education and training“). Beispielhafte Kriterien: Ausmaß der Forschung Forschungskonzeption, Umweltbeobachtungs- oder Monitoningsysteme, Datenmanagement, Partizipation, Forschungsinfrastrukturen, Nutzung als Freilandlabor, Beachtung der MAB-Forschungsschwerpunkte und -vorgaben, Ausmaß des Nutzens aus der Forschung für die Region, Entwicklung von Modellen und Methoden, Aus- und Fortbildungsprogramme, Informationszentrum, Umveltbildungseinnichtungen und -aktivitäten, Demonstrationsobjekt, Informationsmaterialien, etc.

- Implement the Biosphere Reserve conoept (,implementation of Biosphere Reserve concept"). Beispielhafte Kriterien: Integration und Abstimmung aller regionalen Aktivitäten und Funktionen (Rahmenkonzept, Managementplan), transnationale Kooperationen, Zonienung, Partizipation, Interdisziplinarität, Strategien zur Mittelbeschaffung aus Wirtschaft oder Nichtregienungsorganisationen, Evaluienungsmechanismen (Sevilla-Strategie), Public relations, etc.

Die den einzelnen Zielen zugeordneten Indikatoren, die in Summe das Ergebnis darstellen, leiten sich aus dem Fragebogen (vergl. Anhang-3) bzw. dem Indikatorenset der Sevilla-Strategie ab (vergl. UNESCO 1996). Um einen Achsenwert zu ehalten, wurden zunächst die „Nein“-Antworten mit den "Ja"-Antworten des Fragebogens in Beziehung gesetzt (es wurden nur die sinnvollen, wertenden Fragen zur Auswertung herangezogen). Aufgnund der unterschiedlichen Anzahl an Fragen pro Ziel war es notwendig, die „Nein"-Antworten auf den Wert $1 \mathrm{zu}$ standardisieren und die "Ja"Antworten auf den entsprechenden Verhältniswert zu relativieren. Nur so kann eine übersichtliche Darstellung im Diagramm erfolgen. Da es sich bei den Indikatoren meist um unscharfe Mengenzugehönigkeiten und Regeln handelt, wurden in einem zweiten Schnitt unter Einbeziehung der Literaturauswertung und nach eigener Einschätzung die Stellgrößen 
nachjustiest. Bei einem derartigen Modell ergeben sich unvillkürdich Grauzonen. Sie wurden in einem dritten Schnitt in Form einer Rückkoppelungsnunde mit den Experten vor Ort nochmals eingeengt. Das Ergebnis ist ein Gesamtbild, in dem die Summe aller Eingabeparameter als integrative Größe sichtbar wird.

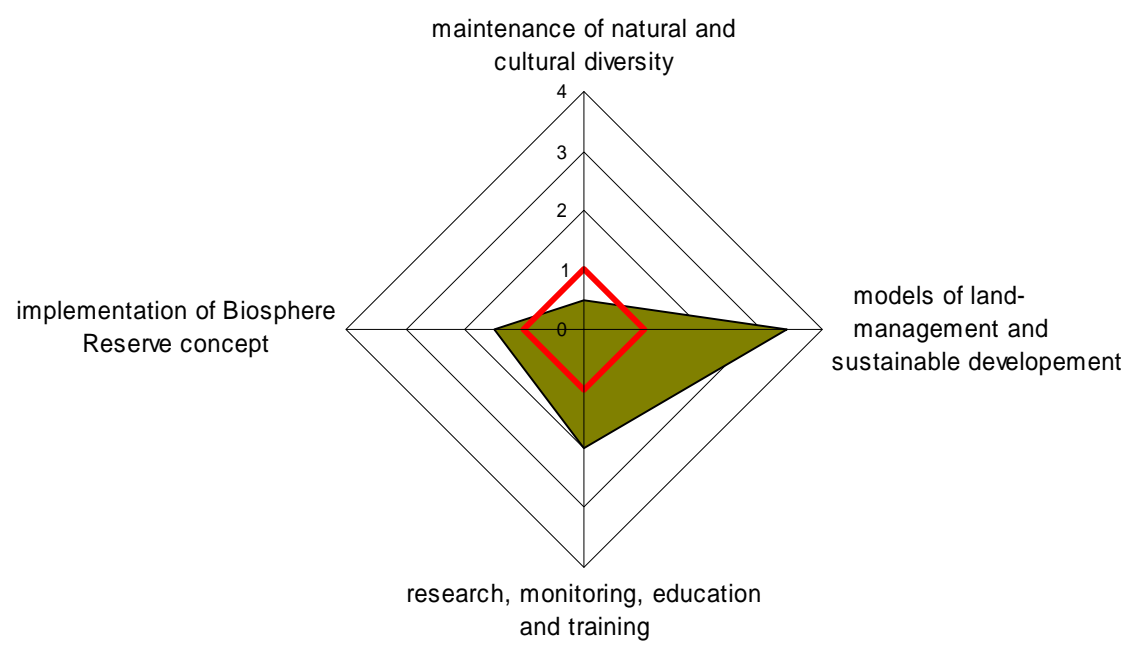

Abbildung 14: Beispidsdiagrammzur V isualisienungdar ZidemeidhunggemäßS Seilla-Strateje

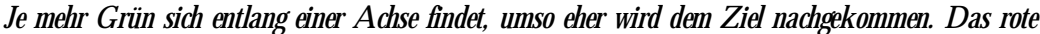
Rechtek stelt dabei einen Schmelenmat dar, dbehalb dessen die SeillaTaugidkeit in Summe positiv geschen weden kann DieReduktion ener Rahmenidhtinieauf dievedidtteleInformation die durch vier Adneen abghildat vird vedang, dass jedes Teilzid noch einer gsandaten Beradhung untezogen waden (veg. Ausfiiknungen $\mathrm{zu}$ den Erghonissen der einzdnen Biosphärenparks).

Die Interviews und die Auswertungen sind vor folgendem Hintergnund zu sehen:

- Auswahl der Intenviempartner für Expenteninterviews: In Absprache mit dem Auftraggeber (Östemeichisches MABNationalkomitee, Prof. Grabherr) ururden die jeweiligen
Biosphärenparkmanager als goeignete Interviewpartner ausgewählt Sie besitzen naturgemäß den besten Einblick in das Geschehen im Biosphärenpark. Eine gewisse Befangenheit bei der Beantwortung der Fragen kann dabei allerdings nicht ausgeschlossen werden.

- Nationalpark als dominante Schutzkategorie: Die Biosphärenparks Lobau und Neusiedler See werden von der Schutzkategorie Nationalpark überlagent. Beide Biosphärenparkbeauftragten sind dabei auch wesentlich in die Agenden des jeweiligen Nationalparks eingebunden. Die Antworten sind daher vor dem Hintergnund dieser Doppelfunktion zu sehen, was eine eindeutige Zuordnung der Aussagen $\mathrm{zu}$ den Rubriken „Biosphärenpark" oder „Nationalpark" erschwert. Es wird daher z.T. versucht, die Beurteilung zum einen mit strengem Bezug auf Biosphärenparkaktivitäten, zum anderen unter Berücksichtigung der Nationalparkaktivitäten, getrennt zu beleuchten.

- Visualisienung der Kemaussagen: Die Diagramme sollen in Art, Herkunft, Qualität und Quantität äußerst heterogene Daten auf einen Blick erfassbar machen. Ihnen liegt somit keine oder nur teilweise statistische Genauigkeit zugnunde, vielmehr findet eine starke Verdichtung der Eingangsparameter statt (Komplexitätsreduktion auf ein Modell). Informationsverluste sind dabei unausweichlich. Die gewählte Form der Visualisienung wird jedoch als goeignetes Mittel angesehen, um Kemaussagen übersichtlich darzustellen.

- Eindringtiefe: Die gewählte Eindringtiefe ist vor dem Hintergnund der nur ansatzweise implementierten Biosphärenparkidee, dem damit einhergehenden Manko an Unterlagen und Anknüpfungspunkten und fehlenden auf Österreich abgestimmten Evaluienungsmethoden zu sehen. $\mathrm{Zu}$ detailliente Aussagen hätten nur beispielhaften oder zufälligen Charakter gehabt. Ziel war aber die Generienung einer Gesamtsicht, die kare, nichtungweisende Aussagen über grundsätzliche und dringlich zu behandelnde Eckpunkte/ Basisfunktionen/ Enfordemisse treffen lässt.

- Evaluienung der Ergebnisse: Aus den Unschärfen dervorhandenen Daten und subjektiven Ansichten der involvierten Personen eine 
fundierte Gesamtsicht mit dem Anspruch wissenschaftlicher Genauigkeit zu erstellen, ist mit gewissen Unsicherheitsgraden behaftet. Es wird daher folgender Ansatz gewählt: „Data don't have to be exact, but all have to agree upon". Gegen Projektende werden daher die im Projekt involvienten Personen gebeten, den Berichtsenturuf nochmals mit ihren Ansichten abzugleichen.

- Benücksichtigung untenschiedlicher Entwickhungsstufen: Die Bewertung hat den unterschiedlichen Entwicklungsstufen, in denen sich die Biosphärenparks befinden, Rechnung zu tragen. Eine ähnliche Ausprägung eines Kriteriums in zwei Biosphärenparks kann daher trotzdem zu unterschiedlicher Bewertung führen.

- Individuelle Entstehungsgeschichte: Das Gutachterteam ist sich dessen bewusst, dass die Beurteilungsmethode mittels Sevilla Kriterien vor dem Hintergnund der unterschiedlichen Entstehungsgeschichten zu sehen ist. Die Biosphärenparks der ,alten Generation" besitzen im Vergleich zu den Biosphärenparks der „neueren Generation“ entscheidende Nachteile. Ihre Entstehung wurde aber von anderen, unter den damaligen Verhältnissen als angemessen erscheinenden Rahmenbedingungen begleitet. Es kann daher nicht vorausgesetzt verden, dass durch die erst 1995 eingeführte Sevilla-Strategie gewachsene Strukturen derart kurzfristig angepasst oder ersetzt werden können. Nichtsdestotrotz soll durch die Bewertung die IST-Situation klar dargestellt werden, ohne jedoch näher auf die nachvollziehbaren und oft allzu verständlichen Beweggründe/ Hintergründe (wie eben zB. die individuelle Entstehungsgeschichte) einzugehen. Den Aussagen wohnt daher ein bestimmtes Potenzial an "Provokation“ oder „Plakativität“ inne. Es wurde aber letztendlich als sinnvoller erachtet, die Beurteilung nach dem Schema "Schwarz" oder "Weiß" vorzunehmen, als durch ein allzu detailliertes und somit diffuses Bild den Blick aufs Wesentliche zu verstellen. 